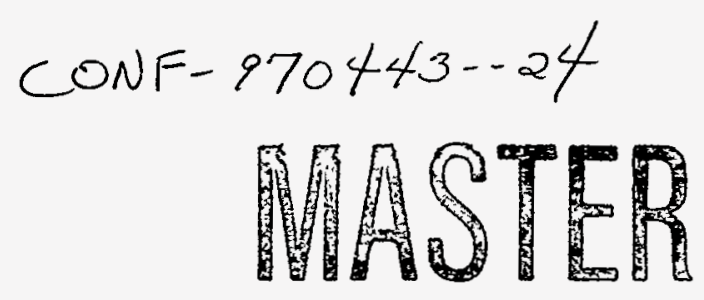

\title{
The n-particle Picture and the Calculation of the Electronic Structure of Atoms, Molecules, and Solids
}
A. Gonis; T. C. Schulthess; P. E. A. Turchi; and J. van $\mathrm{Ek}^{\S}$
RECEIVED
JUL 281999

\section{Introduction}

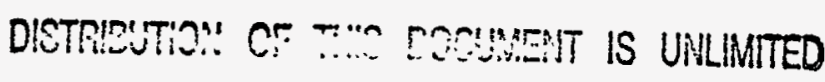
OST

There are several problems in the physics of quantum systems whose importance is attested to by the time and effort that have been expended in search of their solutions. A class of such problems involves the treatment of interparticle correlations with the electron gas in an atom, a molecule (cluster) or a solid having attracted significant attention by quantum chemists and solid-state physicists. This has led to the development of a large number of theoretical frameworks with associated computational procedures for the study of this problem. Among others, one can mention the local-density approximation (LDA) to density functional theory (DFT) $[1,2,3,4,5]$, the various forms of the Hartree-Fock (HF) approximation[1, 2, 6, 7], the so-called GW approximation $[8,9,10]$, and methods based on the direct study of two-particle quantities $[11,12,13]$, such as two-particle reduced density matrices[14, 15, 16, 17, 18], and the closely related theory of geminals[17, 18, 19, 20], and configuration interactions (CI's)[21]. These methods, and many of their generalizations and improvements[22, 23, 24] have been discussed in a number of review articles and textbooks $[2,3,25,26]$.

The great formal variety that characterizes these methods provides evidence of the complexity of the problem at hand. This diversity has evolved

-Department of Chemistry and Materials Science, L-268, Lawrence Livermore National Laboratory, Livermore, California, 94550, USA

$\dagger_{2}$. Metals and Ceramics Division, Oak Ridge National Laboratory, Oak Ridge, TN 37831, USA

†Department of Chemistry and Materials Science, L-268, Lawrence Livermore National Laboratory, Livermore, California, 94550, USA

${ }^{\S}$ Department of Physics, Tulane University, New Orleans, LA 70118, USA

- The submitted manuscript has been authored by a contractor 
out of attempts to treat specific features of this complex problem, ranging from the electron gas in "small systems", e.g., atoms and molecules, and atomic clusters, where HF-based methods and CI's, for example, have been found very useful, or electrons in solids where the LDA has met with considerable success in determining ground-state densities and energies. Even a cursory review of the advantages and limitations of the various methods that have been proposed to study the inhomogeneous electron gas is far beyond the scope of the present work. Suffice it to say that each of these methods have come to be accepted as the most appropriate for a particular aspect of this very complex problem, while having only limited and even questionable applicability in other domains.

However, in spite of much progress along different directions and in different areas, there exists no unified conceptual framework[26] capable of providing systematic improvements to the study of correlations across a broad spectrum of materials types, while possessing well-defined convergence characteristics. Furthermore, the disparity between so-called single-particle methods, such as the LDA, and many-body techniques, e.g, Green-function theory, has led to the perception that these two approaches are irreperably divorced and disjoined from one another in a formal sense.

This perception, however, may be premature. It appears that it is possible to adopt a point of view which provides for a unified approach to the problem of correlations for systems of any size or structure. This viewpoint is the natural outgrowth of work initiated as far back as the 1930's and which has continued in certain areas to the present.

Initially, Pauling used the concept of a single state (bond state) to describe the behavior of two electrons participating in molecular binding[27]. In the late 1950's and early 1960's, Brueckner[11] and collaborators, and Kadanoff and Baym[12, 28] showed, respectively, how the two-particle tmatrix and the two-particle Green function could be used in the study of interacting quantum systems in a straightforward generalization of the methods based on the corresponding single-particle quantities. However, the most telling developments have accrued from a particular approach initiated in the field of atomic and molecular physics. In this methodology, it has proved profitable to study the electronic structure and related properties of atoms by considering all the $N$ electrons in the system as forming a single particle in a space of $3 N$ dimensions. There is a large body of work on this approach, from which a single representative reference is given here[29].

In this method, the problem of $N$ particles in 3-dimensional space is mapped onto a problem of a single-particle in a hyperspace of $3 \mathrm{~N}$ dimensions, moving under the influence of an external potential. This potential is made up of the external potentials acting on the particles, as well as their 
mutual interactions. This reduction to a single-particle in an external field has the formal nicety of allowing the use of single-particle methods in the solution of a many-particle problem. Not to downplay the significance of the computational ramifications of this formalism, we note that applying singleparticle methods to hyperspace, e.g., partial-wave analysis of scattering, can be an extremely difficult task. In fact, it becomes quickly intractable when the number of particles increases beyond even some fairly small number, say $N=4$. This feature has limited the application of this method to fairly small systems such as the He atom and the molecule $\mathrm{H}_{2}^{+}$, (where the two particles combined into one in a space of six dimensions are the nuclei). Applications to extended systems characterized by a large number of centers of force seems to be hopelessly out of reach. (However, for some innovative approaches to atoms with infinite charge and the interpretation of the results, the reader is referred to the literature[29].)

The works referred to above indicate the usefulness of viewing an $\mathrm{N}$ particle system from a higher-dimensional pespective. In doing so, one should attempt to strike a balance between conceptual clarity and computational efficiency, which mitigates against considering calculations in $3 n$-dimensional space except for rather small values of $n$. It appears that such a procedure may be profitably employed if a system of $N$ particles were to be considered as consisting of a collection of units or sets, $\left\{I_{k}\right\}$, each containing $n_{k}$ particles, so that

$$
\sum_{k} n_{k}=N .
$$

The resulting problem associated with these sets of particles that interact with one another is obviously formally identical to the original one (only the meaning of the term particle having been redefined in the present case). However, it possesses the formal advantage of allowing, in principle, the systematic approach to an exact solution by treating the entire system as a single unit ( $N$-particle picture). The operative words here are "in principle", as practical applications do not seem to be possible but for the smallest number of particles in a unit, say $n=2$ or $n=3$. However, in such an implementation, the interparticle correlation is treated directly and explicitly within a unit, resulting in a more accurate treatment of the system the larger the number of particle in a unit. Furthermore, this methodology can be applied to a broad spectrum of systems, such as atoms, molecules, and solids, with fairly strong expectations that it will yield improved results compared to its corresponding use within strictly single-particle methods. For example, one can determine the ground-state two-particle, corralated density and energy in terms of two-particle states by using a generalization of DFT and LDA to the appropriate hyperspace, as is discussed below. Similarly, al- 
loy phase stability and transport properties (which can be expressed directly in terms of the two-particle Green function) can also be studied, as can the ground state properties of small clusters, molecules, and atoms, within appropriate generalizations of the HF method. Finally, this method may provide a more realistic description of the electronic structure of a system than can be achievd within a single-particle framework. Even if its computational implementation is only possible for small values of particles in a unit, the method to be developed below has one very important conceptual characteristic: it tends to obscure the sharp boundaries that divide singleparticle (band-theory) methods from many-body approaches, leading to a broader viewpoint and providing a possibly deeper insight into the effects of correlations than has been hitherto possible.

\section{Basic Notions}

We begin with the Hamiltonian for a system of $N$ interacting particles,

$$
\begin{aligned}
H & =\sum_{i}-\nabla_{i}^{2}+\sum_{i} v_{i}+\frac{1}{2} \sum_{i \neq j} v_{i j} \\
& =\sum_{i} h_{i}+\frac{1}{2} \sum_{i \neq j} v_{i j} .
\end{aligned}
$$

Here, $\nabla_{i}^{2}$ is the kinetic energy operator for particle $i$, (with $\hbar=1,2 m_{i}=$ 1 , for all $i), v_{i}$ represents the interaction of particle $i$ with an external potential, such as that associated with nuclei in the system, and $v_{i j}$ represents the mutual interaction between particles $i$ and $j$. The definition of the singleparticle Hamiltonian, $h_{i}$, is evident. We are interested in the solutions of the many-particle time-independent Schrödinger equation,

$$
H \Psi=E \Psi,
$$

where $\Psi$ is the many-particle wave function for the system.

At least two different possible viewpoints present themselves immediately as alternative procedures for solving Eq.(3). One may consider the entire interacting system as a single particle in a configuration space of $3 N$ dimensions, or one may consider the system wave function as consisting of certain combinations of wave functions of individual particles. In either case, of course, one is obligated to incorporate into the solution the statistics, either Fermi or Bose, governing the behavior of the system.

The first viewpoint defines the $N$-particle picture and leads in principle to an exact solution of Eq.(3). Leaving aside the question of whether or 
not one could extract useful information from the complicated form that such an exact solution for large values of $N$ would be bound to take, the computational hindrance associated with this approach as $N$ increases has mitigated against widespread use of such a direct procedure. At the same time, where it has been implemented[29], such as in connection with the $\mathrm{He}$ atom, this approach has yielded results which agree to great precision with those of exact numerical procedures.

The second viewpoint defines what will be referred to as the single-particle picture, in which one attempts the construction of the many-body solution of Eq.(3) through the use of solutions associated with individual particles. It should be emphasized that in the language used here, the single-particle picture is not synonymous to what has come to be known as single-particle theory, in which the properties of the entire system are studied through judiciously chosen single-particle solutions of an effective Schrödinger equation. In the present terminology, the methods of single-particle theory, as well as canonical many-body theory are developed within the single-particle picture as defined above. Not to belabor the possibly obvious point, we should note that the $\mathrm{N}$-particle picture mentioned above lends itself to computational procedures which are themselves generalizations of methods developed within single-particle theories. In any case, because of relative ease of implementation, the study of interacting quantum systems has proceeded almost exclusively within the single-particle picture.

Unlike the $N$-particle picture, which in principle leads naturally to the exact solution of the many-particle Schrödinger equation, the single-particle picture has led to the development of a number of different approximation schemes designed to address particular issues in the physics of interacting quantum systems. A particularly pointed example of this state of affairs is the strict dichotomy that has set in between so-called single-particle theories and canonical many-body theory[30,31]. Each of the two methodologies can claim a number of successful applications, which tends to reinforce the perceived formal gap between them.

Under these conditions, one is moved to ask if indeed this gap is real or perhaps could be bridged in a way that may reveal the unified features of the problem and possibly lead to a deeper understanding of the behavior of interacting quantum systems. The work described here is an attempt to bridge this gap in a coputationally viable way. It is based on a point of view bounded by the single-particle picture at one end and the $N$-particle picture at the other. In this point of view an interacting quantum system of $N$ particles is taken to consist of non-overlapping units or sets of particles, $I_{k}=\left\{\mathbf{r}_{1}^{I_{k}}, \mathbf{r}_{2}^{I_{k}}, \cdots, \mathbf{r}_{k}^{I_{k}}\right\}$, so that a particle belongs to only one such unit, and the particles in all these sets equal $N$, the total number of particles in 
the system. Denoting the number of particles in a set $I_{i}$ by $n_{i}$, leads to Eq.(1). At this point, there is no restriction as to the number of sets chosen or the numbers of particles in each set. This seems to raise the danger of possibly mishandling the statistics of the system by treating some particles in a preferrential way. It will be shown below that, even though this is a "real" danger, it can be avoided.

With these considerations in mind, we return to the Hamiltonian of Eq.(2). If we denote the Hamiltonian describing the particles in a unit or subsystem $I_{k}$ by $H_{I_{k}}$,

$$
H_{I_{k}}=\sum_{i \in I_{k}} \nabla_{i}^{2}+\sum_{i \in I_{k}} v_{i}+\sum_{i \in I_{k}, j \in I_{k}} v_{i j}
$$

we can write the Hamiltonian of the original system in the form,

$$
H=\sum_{k} H_{I_{k}}+\frac{1}{2} \sum_{k \neq l} v_{I_{k}, I_{l}}
$$

where

$$
v_{I_{k}, I_{l}}=\frac{1}{2} \sum_{i \in I_{k}, j \in I_{l}} v_{i j}, I_{k} \neq I_{l}
$$

where $i \in I$ indicates that $i$ belongs to the set $I$ and the last expression defines the mutual interaction of particles across two different units.

Now, the Hamiltonian in the form of Eq.(5) looks formally identical to that ${ }^{-}$in Eq.(2), with the sets of particles $I_{k}$ in the new description playing the role of individual particles in the original (single-particle) picture. We will refer to this point of view as the $n$-particle picture. It is seen that the traditional single-particle picture corresponds to $n=1$, while the $N$ particle picture is obtained when $n=N$, the total number of particles in the system. Therefore, if we treat the Hamiltonian of Eq.(5) as consisting of individual "particles" in appropriately defined higher-dimensional spaces, we go a long way toward obtaining the unifying viewpoint alluded to above. In fact, however the Hamiltonian of Eq.(5) may be treated, and two different ways are presented below, one is assured of an exact limit as the number of units or subsystems approaches one and, correspondingly, $n \rightarrow N$.

There are, however, some very important issues still to be addressed before specific techniques are introduced for solving the Schrödinger equation in connection with the form of the Hamiltonian of Eq.(5). These have to do with retaining the proper symmetry in the wave function for the system as a whole when written in terms of wave functions of the higher-dimensional particles on which the $n$-particle picture is based. In the following, we shall use 
the generic term "particle" to refer either to an ordinary particle in three dimensions, or to single point in a space of $3 n$ dimensions, $\mathbf{x}_{n}=\left(\mathbf{r}_{1}, \mathbf{r}_{2}, \cdots, \mathbf{r}_{n}\right)$, describing $n$ ordinary particles. It is hoped that the context of the discussion will provide sufficient indication as to what is meant to avoid confusion.

Let us first consider the case of distinguishable particles and take the wave function of the system, $\Psi\left(r_{1}, r_{2}, \cdots, r_{N}\right)$, to be a product of single-particle states. Denoting these states by $\Phi_{I_{k}}\left(\{\mathbf{r}\}_{I_{k}}\right)$, where $\{\mathbf{r}\}_{I_{k}}$ denotes the set of coordinates of the ordinary particles assigned to $I_{k}$, we have

$$
\begin{aligned}
\Psi & =\Pi_{k} \Phi_{\alpha_{k}}\left(\{\mathrm{r}\}_{I_{k}}\right) \\
& =\Pi_{k} \Phi_{\alpha_{k}}\left(\mathrm{x}_{I_{k}}\right),
\end{aligned}
$$

where $\alpha_{k}$ is a state of the particle $I_{k}$. These states can be chosen to be both orthonormal and complete in the usual sense. These states can be found by the usual procedure of minimizing the quantity,

$$
I=\langle\Psi|H| \Psi\rangle-\sum_{k} \epsilon_{\alpha_{k}} \int \mathrm{d}^{3 n} x_{I_{k}}\left|\Phi_{\alpha_{k}}\left(\mathrm{x}_{I_{k}}\right)\right|^{2}
$$

with respect to $\Phi_{\alpha_{k}}^{*}\left(\mathbf{x}_{I_{k}}\right)$, where an asterisk denotes a complex conjugate. This leads to an eigenvalue equation determining the levels $\epsilon_{\alpha_{k}}$,

$$
\left[H_{I_{k}}+v^{\mathrm{eff}}\right] \Phi_{\alpha_{k}}=\epsilon_{\alpha_{k}} \Phi_{\alpha_{k}}
$$

where $v^{\text {eff }}$ denotes the interaction of the particles in $I_{k}$ with an external field, (e.g., the nuclei in the system), as well as their interactions with the particles in other units, $I_{l} \neq I_{k}$. This term takes the form of a Hartreelike expression involving the density. of the particle $I_{k}$, and reduces to the conventional Hartree expression in the canonical single-particle description.

We now consider the case of indistinguishable particles, treating explicitly a system of Fermions. It is convenient to write the wave function for a system of free Fermions in the form,

$$
\Psi_{i_{1} i_{2} \cdots i_{N}}^{0}\left(\mathbf{r}_{1}, \mathbf{r}_{2}, \cdots, \mathbf{r}_{N}\right)=\left[\begin{array}{llll}
i_{1} & i_{2} & \cdots & i_{N} \\
\mathbf{r}_{1} & \mathbf{r}_{2} & \cdots & \mathbf{r}_{N}
\end{array}\right]
$$

In this notation, we designate a single-particle state by the symbol $\phi_{i}(\mathbf{r})=\left[\begin{array}{l}i \\ \mathbf{r}\end{array}\right]$. This notation is meant to emphasize the fact that a fully antisymmetrized wave function for non-interacting particles may be obtained by distributing individual particles over all possible states written in some order or, alternatively, by distributing all possible states over particles which 
have been labeled in a permanent way. Thus, a fully antisymmetrized wave function is obtained from the last expression as a sum of terms resulting from keeping the particle labels in the order given in the lower row, and performing all possible permutations on the state indices in the top row. From now on, we take this expression to denote this fully antisymmetrized wave function.

Let us now partition the particles in the system according to some scheme which assigns $n_{i}$ particles to distinct sets $I_{i}$ in accordance with Eq.(1). As this partitioning depends only on the number of particles in each unit, it can be effected by dividing the lower row in the experession for the wave function, Eq.(10), into sets of $n_{i}$ particles beginning from the left. Thus, particles 1, 2, $\ldots, n_{1}$ are taken to form the first set, particles $n_{1}+1, n_{1}+2, \ldots, n_{1}+n_{2}$, to form the second set, and so on. Each set is marked off by means of vertical lines as given in the following expression written explicitly for the case of pairs, (assuming that the total number of particles is divisible by two),

$$
\begin{aligned}
& {\left[\begin{array}{l|l|l|l}
i_{1} i_{2} & i_{3} i_{4} & \ldots & i_{N-1} i_{N} \\
\mathrm{r}_{1} \mathrm{r}_{2} \mid & \mathrm{r}_{3} \mathrm{r}_{4} \mid & \ldots & \mathrm{r}_{N-1} \mathrm{r}_{N}
\end{array}\right]=\hat{A}\left[\begin{array}{cc}
i_{1} & i_{2} \\
\mathrm{r}_{1} & \mathrm{r}_{2}
\end{array}\right] } \\
\times & {\left[\begin{array}{ll}
i_{3} & i_{4} \\
\mathrm{r}_{3} & \mathrm{r}_{4}
\end{array}\right] \cdots\left[\begin{array}{cc}
i_{N-1} & i_{N} \\
\mathrm{r}_{N-1} & \mathrm{r}_{N}
\end{array}\right], }
\end{aligned}
$$

where the antisymmetrizing operator, $\hat{A}$, acts on the state indices, and the vertical lines indicate the partitioning of the system into pairs of particles and their corresponding pair states. In this expression, we can use the combined notation $\mathrm{x}_{k}$ to denote the particles in $I_{k}$ as a single point in a space of $3 n_{k}$ dimensions. In the present case, we have $x_{1}=\left(r_{1}, r_{2}\right), x_{2}=\left(r_{3}, r_{4}\right)$, and so on. It is, therefore, seen that because of the equivalence of distributing states over particles to distributing particles over states, the wave function of a system of free Fermions is not affected by a partitioning into sets of specific particles.

To proceed with formal developments, we now associate with each particle $I_{k}$ in hyperspace a coordinate $\mathbf{x}_{k}$, and a state $\alpha_{k}$, which in the form employed here is such as to correspond to all possible independed combinations of the free particle states into the units $I_{k}$. As long as the particles remain free, a fully antisymmetrized wave function can be obtained by antisymmetrizing with respect to particle indices a product of the type given in Eq.(7).

It is tempting to think that this procedure can be used when the states $\alpha_{k}$ are obtained directly through the solution of an appropriate Schrödinger equation in $3 n_{k}$-dimensional space. This, however, is not the case. As a specific example, let us consider the Be atom whose ground state configuration is $1 s^{2} 2 s^{2}$. The states involved are (in a hopefully obvious notation), $\chi_{1}=1 s \uparrow$, 
$\chi_{2}=1 s \downarrow, \chi_{3}=2 s \uparrow$, and $\chi_{4}=2 s \downarrow$. Using these wave functions, an approximate wave function for the system in the form of a Slater determinant can be obtained by antisymmetrizing the product $\chi_{1}(1) \chi_{2}(2) \chi_{3}(3) \chi_{4}(4)$ either with respect to state or particle indices. However, suppose that we are given states of pairs of particles, (each fully antisymmetrized and consistent with the Pauli principle), and choose the two of the lowest energies to construct the ground-state wave function of the system. In this case these would be determinantal wave functions denoted by $\left(\chi_{1}(1) \chi_{2}(2)\right)$ and $\left(\chi_{1}(3) \chi_{4}(4)\right)$, since the state of immediately higher energy than the ground state of two particles is obtained by exciting one of them to the next level. Now, forming the product of these two states and antisymmetrizing with respect to particle indices does not cause the final wave function to vanish for violating the exclusion principle, and thus this incorrect state is not excluded from the final wave function as it should be. This feature is obviously retained when the pair states are obtained directly, rather than as products of single-particle states. Because of it, the ordinary theory of geminals, depending on the determination of two-particle states, does not allow the construction of a properly antisymmetrized wave function that is consistent with the Pauli exclusion principle. Hence, the well-known absence of an aufbau prinzip (building-up principle) in terms of independently determined $n$-particle states. Of course, antisymmetrization with respect to single-particle-state indices would take care of the problem, and eliminate inappropriate wave functions, but this procedure is not viable when $n$-particle states are determined directly and independently of one another.

One of the applications of the $n$-particle picture made below involves the generalization of the Hatree-Fock approximation to $n$-particle states. If one attempted to determine $n$-particle states directly from the equations, coupled with an antisymmetrization procedure with respect to particle indices, one would encounter precisely the same difficulty as noted above. Instead, the problem must be approached from a different perspective.

In this approach, we consider the evolution of a system of particles described by means of the generalized HF equations as the interparticle interaction is turned on, starting from a single Slater determinant. The determinantal state corresponding to the zero-interaction limit provides an "initial condition" for solving the generalized $\mathrm{HF}$ equations within the $n$-particle picture. The states which evolve out of this procedure are known to satisfy the Pauli principle in the zero-interaction limit, and the generalized HF procedure to be described below maintains the correct symmetry as well as the requirements of the exclusion principle.

We also discuss the generalization of density-functional theory to $n$ partical states, $n \mathrm{DFT}$, and the possible extension of the local density ap- 
proximatiom, $n$ LDA. We will see there that the difficulty of describing the state of a system properly in terms of $n$-particle states presents no formal difficultie since DFT is directed only at the determination of the particle density rather than individual-particle wave functions. The extent to which practical applications of $n$ DFT within a generalized Kohn-Sham scheme will provide a viable procedure is commented upon below.

\section{Generalization of the Hartree-Fock Ap- proximation}

The equations of the HF approximation are derived by taking expectation values of the Hamiltonian with respect to a determinantal wave function written in terms single-particle states, incorporating the orthonormality condition by means of a Lagrange multiplier, and minimizing the expression $\left\{\langle\Psi|H| \Psi\rangle-\sum_{i} \epsilon_{i} \int \mathrm{d}^{3} r\left[\begin{array}{l}i \\ \mathbf{r}\end{array}\right]^{*}\left[\begin{array}{l}i \\ \mathbf{r}\end{array}\right]\right\}$ with respect to $\left[\begin{array}{l}i \\ \mathbf{r}\end{array}\right]^{*}$. In the coordinate representation, this yield the "eigenvalue" equation

$$
\begin{gathered}
{\left[-\nabla^{2}+v(\mathrm{r})+\sum_{j \neq i}^{N / 2} \int \mathrm{d}^{3} r^{\prime} \frac{\left|\phi_{j}\left(\mathrm{r}^{\prime}\right)\right|^{2}}{\left|\mathrm{r}-\mathrm{r}^{\prime}\right|}\right] \phi_{i}(\mathrm{r})} \\
-\sum_{j \neq i}^{N} \int \mathrm{d}^{3} r^{\prime} \frac{\phi_{j}^{*}\left(\mathrm{r}^{\prime}\right) \phi_{i}\left(\mathrm{r}^{\prime}\right)}{\left|\mathrm{r}-\mathrm{r}^{\prime}\right|} \phi_{j}(\mathrm{r})=\epsilon_{i} \phi_{i}^{*}(\mathrm{r}) .
\end{gathered}
$$

We note the presence of the direct or Hartree term and the exchange term, third term inside the brackets and first term outside, respectively. The term $i=j$ can be included in the direct term since it is canceled by the corresponding inclusion into the exchange term. Therefore, we have the well-known result that the HF approximation does not include self-interaction terms.

We now extend the formalism just summarized to two-particle states. We begin by writing the wave function of the system of $N$ particles as the sum of products of two-particle states each of which possesses the proper symmetry under exchange of ordinary particles, and with the total wave function being fully symmetrized or antisymmetrized with respect to exchange of individual particles. Denoting such a two-particle state by the symbol $\left[\begin{array}{ll}i & j \\ \mathbf{r}_{1} & \mathbf{r}_{2}\end{array}\right]$ we have for the full wave function the expression given in Eq.(11).

We now envision these pair states to evolve out of free and non-interacting plane-wave states as the interaction with the nuclei and between the particles are introduced. This is a natural way of viewing the evolution of a set of 
initially free particles under the action of an external field (e.g. nuclear attraction) and their mutual interaction. Under the action of the external field the particles form a bound system, described approximately through the solutions of the $\mathrm{HF}$ equations. The $n$-particle picture allows an approximate description of the way in which the interaction among the particles now modifies these HF states. Clearly, this description becomes exact as $n \rightarrow$ $N$. At the same time, the solutions for the $n$-particle states should yield a lowering of the total energy of the system compared to the HF method because of the more accurate treatment in the former of correlation effects that would tend to keep the particles apart thus lowering their potential energy.

In the procedure just outlined, the final wave function retains the proper symmetry under exchange of state indices or particle exchange. This wave function, described in more detail below, corresponds to a particular partition of the particles into pairs, and each of the pairs is associated with every possible two- particle state that can be formed by and evolves out of an original set of single-particle free and non-interacting states. Denoting by a zero subscript two-particle states in free space, we have the following orthonormality

$$
\begin{aligned}
& \int \mathrm{d}^{3} r_{1} \mathrm{~d}^{3} r_{2}\left[\begin{array}{ll}
i & j \\
\mathrm{r}_{1} & \mathrm{r}_{2}
\end{array}\right]_{0}^{*}\left[\begin{array}{ll}
k & l \\
\mathrm{r}_{1} & \mathrm{r}_{2}
\end{array}\right]_{0} \\
= & \int \mathrm{d}^{3} r_{1} \mathrm{~d}^{3} r_{2}\left[\begin{array}{ll}
i & j \\
\mathrm{r}_{1} & \mathrm{r}_{2}
\end{array}\right]^{*}\left[\begin{array}{ll}
k & l \\
\mathrm{r}_{1} & \mathrm{r}_{2}
\end{array}\right]=\delta_{i k} \delta_{j l}
\end{aligned}
$$

and completeness

$$
\begin{aligned}
& \sum_{i j}\left[\begin{array}{ll}
i & j \\
\mathrm{r}_{1} & \mathrm{r}_{2}
\end{array}\right]_{0}^{*}\left[\begin{array}{ll}
i & j \\
\mathbf{r}_{1}^{\prime} & \mathrm{r}_{2}^{\prime}
\end{array}\right]_{0} \\
= & \sum_{i j}\left[\begin{array}{ll}
i & j \\
\mathrm{r}_{1} & \mathrm{r}_{2}
\end{array}\right]^{*}\left[\begin{array}{ll}
i & j \\
\mathbf{r}_{1}^{\prime} & \mathrm{r}_{2}^{\prime}
\end{array}\right]=\delta\left(\mathrm{x}-\mathrm{x}^{\prime}\right)
\end{aligned}
$$

relations.

In order to generalize the $\mathrm{HF}$ equations to $n$-particle states, we perform a variational procedure. In this procedure, it is convenient to identify clearly and uniquely the particle space in which the two-particle wave functions are acting. This identification is considerably easier when the particle pairings are uniquely and distinctly defined, as is done in constructing the wave function or in partitioning the Hamiltonian. We can now obtain an eigenvalue-like equation for the two-particle states $\left[\begin{array}{ll}i & j \\ \mathbf{r}_{1} & \mathbf{r}_{2}\end{array}\right]$ by following the same formal 
procedure as in the single-particle case. From the variational condition

$$
\delta\left[\langle\Psi|H| \Psi\rangle-\sum_{i j} \epsilon_{i j} \int d^{6} x\left[\begin{array}{cc}
i & j \\
\mathbf{x}
\end{array}\right]^{*}\left[\begin{array}{cc}
i & j \\
\mathbf{x}
\end{array}\right]\right]=0
$$

with respect to $\left[\begin{array}{cc}i & j \\ \mathbf{x}\end{array}\right]^{*}$ we obtain the equation in the coordinate representation

$$
\begin{aligned}
& {\left[-\nabla_{\mathbf{x}}^{2}+V(\mathbf{x})+\int \mathrm{d}^{6} x^{\prime} V\left(\mathbf{x}, \mathbf{x}^{\prime}\right) \rho_{(i j)}\left(\mathbf{x}^{\prime}\right)\right]\left[\begin{array}{l}
i j \\
\mathbf{x}
\end{array}\right] } \\
+ & \sum_{n m} \int \mathrm{d}^{6} x^{\prime}\left[\begin{array}{l}
n m \\
\mathbf{x}^{\prime}
\end{array}\right]^{*} V\left(\mathbf{x}, \mathbf{x}^{\prime}\right) \\
\times & \left\{\left[\begin{array}{c|l}
i j \mid & n m \\
\mathbf{x} & \mathbf{x}^{\prime}
\end{array}\right]-\left[\begin{array}{l}
i j \\
\mathbf{x}
\end{array}\right]\left[\begin{array}{l}
n m \\
\mathbf{x}^{\prime}
\end{array}\right]\right\}=\epsilon_{i j}\left[\begin{array}{c}
i j \\
\mathbf{x}
\end{array}\right],
\end{aligned}
$$

where the two-particle density, $\rho_{(i j)}(\mathrm{x})$, is given by the expression

$$
\rho_{(i j)}(\mathbf{x})=\sum_{n m \neq i j}\left[\begin{array}{c}
n m \\
\mathrm{x}
\end{array}\right]^{*}\left[\begin{array}{c}
n m \\
\mathrm{x}
\end{array}\right] \text {. }
$$

It should be emphasized that the combinations of "single-particle" indices, such as $i, j$, are to be viewed as a single combined index denoting the pair state that has evolved out of the individual particle states, $i$ and $j$, under the interparticle interaction (and the interaction with an external potential).

The generalized two-particle HF equations are seen to have a structure equivalent to their single-particle counterparts, exhibiting the presence of a direct term, written in terms of the density, and an "exchange" term. As the canonical HF equations, the present expressions do not contain spurious self-interaction terms. However, unlike the single-particle equations, they allow the determination of fully correlated two-particle states removing to this extent the most basic objection to the HF method.

\subsection{Numerical illustration}

We present the resulting calculations of single-particle spectra for four electrons (2 of each spin direction) on a linear ring of four sites. The singleparticle single-band Hamiltonian describing the non-interacting system is defined by its matrix elements in a tight-binding representation,

$$
H_{i j}^{(1)}=\epsilon_{i} \delta_{i j}+t_{i j}
$$


where $\epsilon_{i}$ is an on-site energy, and $t_{i j}$ represents electron hopping from site $i$ to site $j$ and is taken to connect only nearest neighbor sites. In the calculations reported here, we set $\epsilon_{i}=0$ and $t=1.0$. Also, the energies were assigned an imaginary part of $t / 4.0$ for ease of presenting the spectra. Within this picture, a description of interparticle interactions is often given by means of a term $U_{i j} n_{i} n_{j}$ being added to the Hamiltonian of non-interacting particles, where $U_{i j}$ represents the Coulomb repulsion between two electrons on sites $i$ and $j$, and $n_{i}$ is the number operator for an electron on site $i$. Usually, $U$ is taken to be site diagonal and we will also take this to be the case. In a (restricted) HF treatment of this model Hubbard Hamiltonian, the pair of number operators $n_{i} n_{i}$ is decoupled by replacing one of the two operators by its expectation value. This in turn leads to a Hamiltonian of the non-interacting type, Eq.(18), but with all on-site energies displaced by an amount $U\left\langle n_{i}\right\rangle$. In this description the structure of the single particle spectrum remains identical to that for non-interacting particles.

We now generalize the $\mathrm{HF}$ treatment just described to two-particle space. The two-particle space associated with a ring of four sites is a torus of 16 sites and the Hamiltonian describing two non-inetarcting particles has the form,

$$
H_{i j ; k l}^{(2)}=\left[\epsilon_{i}+\epsilon_{j}\right] \delta_{i k} \delta_{j l}+t\left[\left(1-\delta_{i k}\right) \delta_{j l}+\left(1-\delta_{j l}\right) \delta_{i k}\right] .
$$

This describes a single particle on a torus with site energies $\epsilon_{i}+\epsilon_{j}$ (corresponding to two particles on the linear ring being on sites $i$ and $j$ ), and nearest-neighbor hopping $t$. The interaction between the particles is described by the operator $f(U) n_{i} n_{j} n_{k} n_{l}$, where $f(U)$ is a function of configuration. Thus, when the indices $i, j, k, l$ are all different from one another, $f(U)=0.0$. When only two of the indices are identical, $f(U)=U$, and in the case of two different pairs of identical indices, $f(U)=2 U$. In the present version of the $\mathrm{HF}$ approximation, we decouple the four number operators by replacing two of them by their expectation value (average). In this case, we find that the sites along the main diagonal of the torus have their on-site energies shifted by an amount $2 U\left\langle n_{k} n_{k}\right\rangle+U\left\langle n_{k} n_{l}\right\rangle=\frac{5}{4} U$, while the energies on sites off the diagonal are shifted by $U\left\langle n_{i} n_{i}\right\rangle=\frac{U}{4}$. Thus, the two-particle interacting Hamiltonian, which would contain a term $U$ along the main diagonal of the torus, has all on-site energies shifted by the average energy of a pair of opposite-spin electrons on a site.

Figures 1 shows, for $U=10.0$, the single-particle spectra for four electrons on a ring of four sites (half-filled band) obtained in the ordinary version of $\mathrm{HF}$ treatment (restricted HF) (solid lines) and in the two-particle generalization of this approach described above (dashed lines), compared to the exact results (dotted line). The exact results were obtained from the exact Green function 


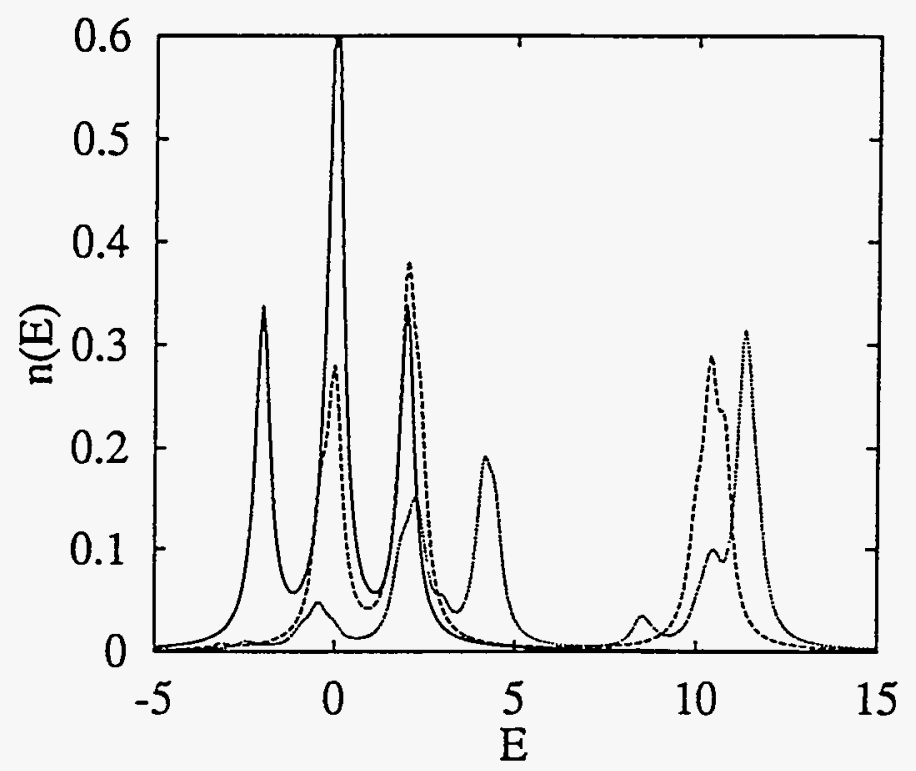

Figure 1: Single-particle spectra for a ring with four sites with $U=10.0$. The solid line is the spectrum calculated from the restricted HF while the dashed line is the spectrum obtained with the $2 \mathrm{HF}$, and the dotted line is the exact spectrum.

of the four-electron system by inverting the full configurational matrix in four-particle space, and integrating out (summing over) the coordinates of three of the particles. Also, for the sake of ease of comparisons, we do not display the shift in the on-site energies since they do not affect the structure of the spectra.

As the present results indicate, the single-particle $\mathrm{HF}$ approximation is completely insensitive to the presence of interparticle interactions. On the other hand, the use of $2 \mathrm{HF}$, the present method, which essentially consists of the treatment of two-particles, provides a much improved representation of the exact spectra.

\section{Generalization of DFT}

Given the formal similarity between the Hamiltonians defined in Eqs.(2) and (5), it follows that the ground-state energy, $E$, is given in terms of a universal functional of the pair (or $n$-particle) density, $n(\mathrm{x})$, which attains its minimum value for the exact pair density. Furthermore, within a Kohn-Sham scheme, the form of this functional is identical to the functional of ordinary DFT but is given in terms of the correlated pair density. The details of this derivation 
are identical to those of ordinary DFT and we confine ourselves to the final result. The energy functional which determines the density in two-particle phase space can be written in the form,

$$
E[n(\mathbf{x})]=T_{\mathrm{s}}[n(\mathbf{x})]+U[n(\mathbf{x})]+E_{\mathbf{x c}}[n(\mathbf{x})],
$$

where $T_{\mathbf{s}}[n(\mathrm{x})]$ represents the kinetic energy of a system of non-interacting particles at the density $[n(\mathbf{x})]$, the quantity $U[n(\mathrm{x})]$ is defined by

$$
\begin{aligned}
U[n(\mathbf{x})] & =\int \mathrm{d}^{6} x V(\mathbf{x}) n(\mathbf{x}) \\
& +\int \mathrm{d}^{6} x_{1} \int \mathrm{d}^{6} x_{2} n\left(\mathbf{x}_{1}\right) V\left(\mathbf{x}_{1}, \mathbf{x}_{2}\right) n\left(\mathbf{x}_{2}\right),
\end{aligned}
$$

and $E_{\mathrm{xc}}[n(\mathrm{x})]$ contains the difference between the exact kinetic energy $T[n(\mathbf{x})]$ and that represented by $T_{\mathbf{s}}[n(\mathbf{x})]$, as well as the difference between the exact interparticle interaction and its "classical" approximation given by Eq.(21),

$$
\begin{aligned}
E_{\mathrm{xc}}[n(\mathrm{x})] & =T[n(\mathrm{x})]-T_{\mathrm{s}}[n(\mathrm{x})]+\int \mathrm{d}^{6} x_{1} \int \mathrm{d}^{6} x_{2} \\
& \times V\left(\mathrm{x}_{1}, \mathrm{x}_{2}\right)\left[n\left(\mathrm{x}_{1}, \mathrm{x}_{2}\right)-n\left(\mathrm{x}_{1}\right) n\left(\mathrm{x}_{2}\right)\right] .
\end{aligned}
$$

Here, $n\left(\mathrm{x}_{1}, \mathrm{x}_{2}\right)$ represents the two-particle correlated density in two-particle space or, equivalently, the correlated density of four ordinary particles. As in ordinary DFT, the exchange-correlation functional is not known but can be used in a local density approximation (LDA) sense through, for example, the study of 6-dimensional jellium.

Both formal analysis and computational developments associated with DFT can be carried over intact to $n \mathrm{DFT}$. For example, the exact twoparticle ground-state density, $n_{0}(\mathrm{x})$, can be determined through a constrained search [32] for that many-particle, properly symmetrized or antisymmetrized wave function, with symmetry imposed with respect to ordinary particles, which yields $n_{0}$ and also minimizes the many-particle energy, $T+V_{\mathrm{pp}}$, where $V_{\mathrm{pp}}$ denotes the interparticle interaction in two-particle space. Essentially any method developed within a single-particle application of DFT for the study of electronic structure can, with appropriate technical modifications, be extended to two-, or $n$-particle states. The use of multiple-scattering theory to calculate fully correlated two-particle densities in solids will be given in a future publication.

\subsection{Numerical illustration}

In order to illustrate the role played by the two-particle density in the determination of the electronic structure, we study the single-particle spectrum of 
four electrons of total spin zero on a four site single-band linear ring described by a Hubbard Hamiltonian

$$
H=\sum_{i} \epsilon_{i} c_{i}^{\dagger} c_{i}+\frac{1}{2} \sum_{i>j} t_{i j} c_{i}^{\dagger} c_{j}+U \sum_{i} n_{i \sigma} n_{i \bar{\sigma}}
$$

where $c_{i}^{\dagger}$ and $c_{i}$ are creation and annihilation operators for an electron on site $i$, the site energies $\epsilon_{i}$ are chosen equal to zero, the hopping term $t_{i j}$ is confined to near neighbors and set equal to 1.0 , and the on-site Coulomb interaction felt by electrons of opposite spin is taken to have the values 1.0 and 10.0, with the respective results shown in Figs. 2 and 3. Exact two-particle and single-particle Green functions and spectra are obtained in down-folding procedure by summing over (integrating out) the coordinates of one and three of the particles, respectively, in the four-particle Green function. This, in turn, is obtained through the usual procedure as a "singleparticle" Green function in a four-particle phase space which in the present case leads to a 256-dimensional matrix. We also include results obtained within a version of the GW approximation in which the self-energy,

$$
\Sigma_{i j}(E)=\lim _{\eta \rightarrow 0} \frac{1}{2 \pi} \int \mathrm{e}^{-\mathrm{i} \omega \eta} W_{i j}(\omega) G_{i j}(E-\omega) \mathrm{d} \omega .
$$

is given in terms of the exact single-particle Green function and the exact screened interaction which is determined in terms of the non-interacting, zero superscript, and exact two-particle Green functions, $W_{i j ; k l}(E)=\left\{G_{i j ; k l}^{(0)}{ }^{-1}-\right.$ $\left.G_{i j ; k l}{ }^{-1}\right\}$.

Finally, we also show the single-particle spectra derived through downfolding from an averaged two-particle Green function in which two-particle states are obtained in the presence of another pair of particles. These states are consistent with the Pauli exclusion principle but neglect the interactions among configurations of two-particle spaces. In all cases the energy was assigned an imaginary part of $0.25 t$ for presentation purposes.

As is seen in these figures, these averaged results are in considerably better agreement with the exact spectra than those obtained in the present version of the GW approximation, particularly for large values of $U$. One also sees that the averaged results overestimate the gap in the strong $U$ limit as may be expected in a non-self-consistent procedure of this type. Even for $U / t=1.0$, a region in which the GW approximation has ben found to give accurate results for real systems, the averaging of the two-particle Green function in the presence of a finite density resolves the band structure much more accurately. We note the resonance at $U=1.0$ which is missed in the GW results, and that both approximate results are displaced somewhat with 


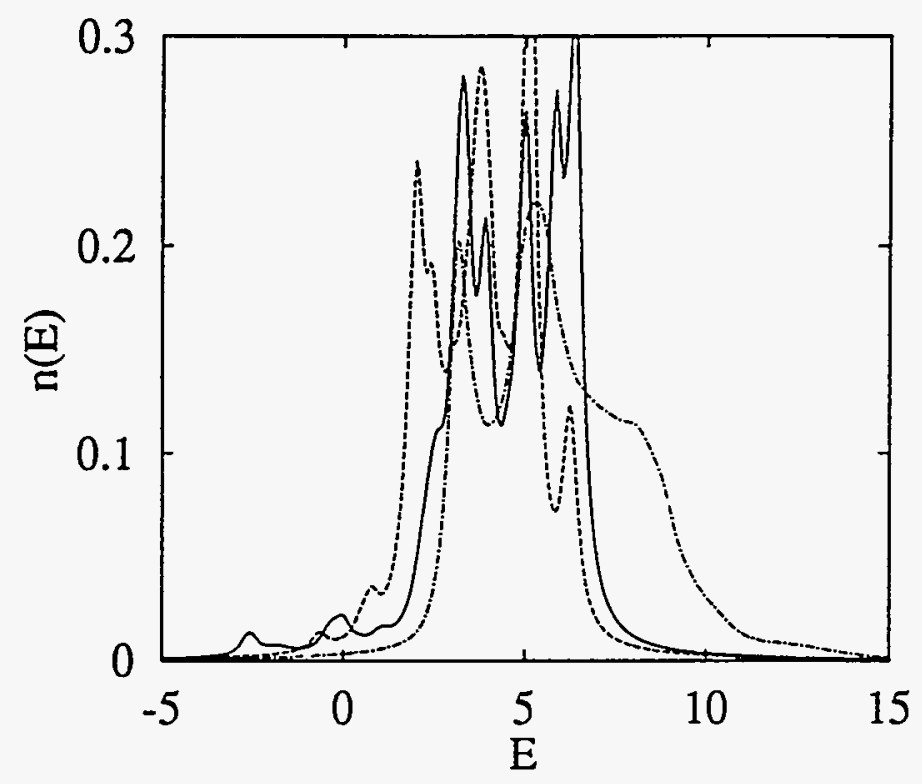

Figure 2: Exact single-particle spectrum (solid line) for four electrons on a four-site ring compared with the results of a GW approximation (dashdotted line) and those obtained from an effective two-particle Green-function (dashed line) for $U=1.0$.

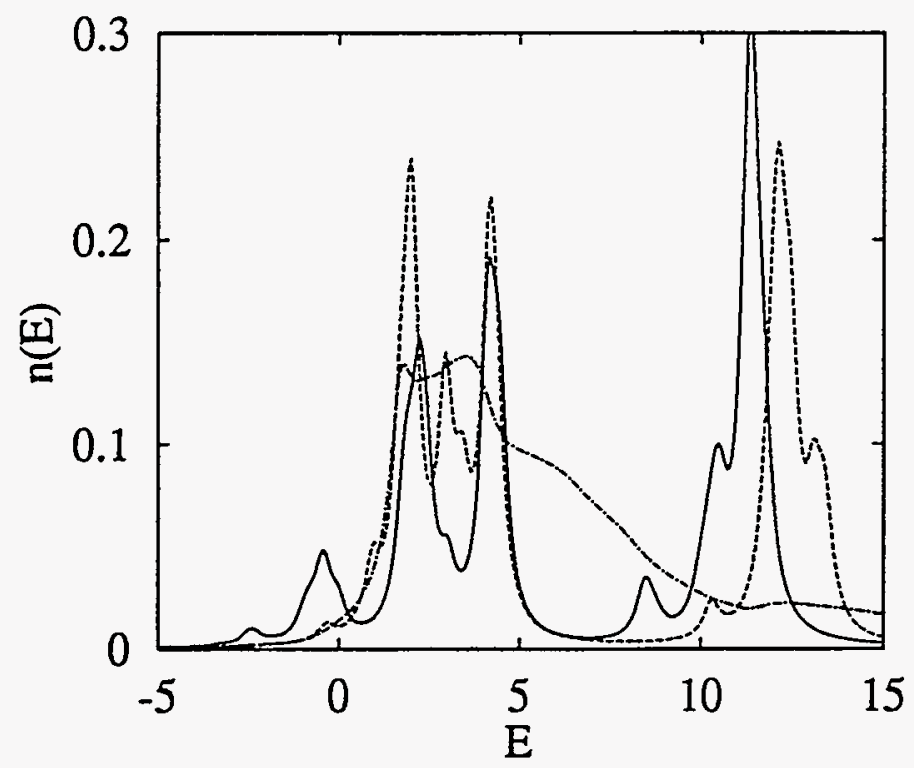

Figure 3: Results analogous to those of the previous figure but for $U=10.0$. 
respect to the exact ones. Much of this effect, along with the wider gap in the stron $U$ limit mentioned above can be traced back to the incomplete treatment of the Pauli principle, i.e., the neglect of configuration interactions. Both of these approximations can be expected to improve with increased dimensionality and lattice connectivity. More detailed discussions of these and other similar numerical results than can be presented here will be given in a future publication.

\section{Discussion}

The formalism presented here indicates the unifying power of the $n$-particle picture and provides an extension of the HF and density functional theories to the treatment of fully correlated two-particle states, (and by a straightforward extension of $n$-particle states). In the case of the HF method, it allows the study of correlation effects in a systematic way in terms of increasing the unit size, becaming exact as $n \rightarrow N$. In the case of DFT, it leads to a general minimum principle governing correlated densities in quantum systems which is analogous to the principle governing single-particle densities. This formalism also becomes exact in the limit in which the entire system of particles is treated as a single unit. Thus, within an $n$-particle picture, $\mathrm{HF}$ theory and DFT are seen to be two different methods leading to exact results in the same limiting procedure.

Although its computational implementation of the method, say within a partial-wave analysis, increases in difficulty rapidly with increasing dimensionality of the $n$-particle phase space, its application to two-particle states is well within the domain of present computational technology.

\section{Acknowledgment}

One of the authors (AG) is grateful to John Perdew, Mel Levy, Kreion Burke, W. H. Butler, and X.-G. Zhang for clarifying discussions. Support for this work was provided by Lawrence Livermore National Laboratory under the auspices of the U.S. Department of Energy under Contract No. W-7405ENG-48. Work at Oak Ridge was supported by the Division of Materials Sciences, U.S. Department of Energy under contract DE-AC05-96OR22464 with Lockheed Martin Energy Research Corporation. 


\section{References}

[1] P. Hohenberg and W. Kohn, Phys. Rev. 136, B864 (1964).

[2] R. G. Par and Weitao Yang, Density Functional Theory of Atoms and Molecules, (Oxford University Press, New York, 1989).

[3] R. M. Dreitzler and E. U. K. Gross, Density Functional Theory, (Springer Verlag, Berlin, New York, 1990).

[4] Theory of the Inhomogeneous Electron Gas, Edited by S. Lundqvist and N. H. March, (Plenum, New York, 1983).

[5] R. O. Jones and O. Gunarson, Rev. Mod. Phys. 61, 689 (1989).

[6] S. Massida, M. Posternak, and A. Baldereschi, Phys. Rev. B48, 5058 (1993).

[7] C. Pisani, R. Dovesi, and C. Roetti, Hartree-Fock Ab Initio Treatment of Crystaline Systems, (Springer-Verlag, Berlin, 1988).

[8] Lars Hedin, Phys. Rev. 139, A796 (1965).

[9] M. S. Hybertsen and S. G. Louie, Phys. Rev. Lett. 55, 1418 (1985); Phys. Rev. B34, 5390 (1986).

[10] R. W. Godby, M. Schlütter, and L. J. Sham, Phys. Rev. Lett. 56, 2415 (1986); Phys. Rev. B37, 10159 (1988).

[11] K. A. Bruckner and C. Levinson, Phys. Rev. 97, 1344 (1955).

[12] Gordon Baym and Leo P. Kadanoff, Phys. Rev. 124, 287 (1961).

[13] Paul Ziesche, Phys. Lett. A195, 213 (1994).

[14] P. -O. Löwdin, Phys. Rev. 97, 1474 (1955).

[15] R. McWeeny, Rev. Mod. Phys. 32, 335 (1969).

[16] A. J. Coleman, Rev. Mod. Phys. 35, 668 (1960).

[17] E. R. Davidson, Reduced Density Matrices in Quantum Chemistry, (Academic Press, New York, 1976).

[18] R. Erdahl and V. H. Smith, eds. Density Matrices and Density Functionals, (Reidel, Dordrecht, 1987). 
[19] A. C. Hurley, Electron Correlation in Small Molecules, (Academic Press, New York, 1976).

[20] W. Kutzerlnigg, Einführung in die Theoretische Chemie, Bd.2, 2nd Ed. (VCH, Weinheim 1994).

[21] Ralph E. Chistoffersen, Basic Principles and Techniques of Molecular Quantum Mechanics (Springer Verlag, New York, 1989).

[22] R. D. Cowan, Phys. Rev. 163, 54 (1967).

[23] J. P. Perdew and G. L. Oliver, Solid State Commun. 34, 933 (1980).

[24] J. P. Perdew and A. Zunger, Phys. Rev. B23, 5048 (1981).

[25] Lars Hedin and Stig Lundqvist, Solid State Physics, (Academic Press, New York, 1969), Supplement 23.

[26] Peter Fulde, Electron Correlations in Molecules and Solids, (Springer Verlag, Berlin, New York 1995).

[27] L. Pauling, The Nature of the Chemical Bond and the Structure of Molecules and Solids, (Cornell University Press, Ithaca, NY 1960).

[28] Leo P. Kadanoff and Gordon Baym, Quantum Statistical Mechanics, (Benjamin, New York, 1962).

[29] John Avery, Hyperspherical Harmonics, (Kluwer, Dordrecht, 1989), and references therein.

[30] Alexander L. Fetter and John Tirk Wallecka, Quantum Theory of Many Particle systems (McGraw-Hill, New York 1971).

[31] E. K. U. Gross and E. Runge, Vielteilchentheorie, (Teubner, Stuttgart 1986).

[32] Mel Levy and John P. Perdew in Density Functional Methods in Physics, edited by Reiner M. Dreizler and Joao da Providencia (Plenum, New York, 1985), p. 11. 


\section{DISCLAIMER}

This report was prepared as an account of work sponsored by an agency of the United States Government. Neither the United States Government nor any agency thereof, nor any of their employees, make any warranty, express or implied, or assumes any legal liability or responsibility for the accuracy, completeness, or usefulness of any information, apparatus, product, or process disclosed, or represents that its use would not infringe privately owned rights. Reference herein to any specific commercial product, process, or service by trade name, trademark, manufacturer, or otherwise does not necessarily constitute or imply its endorsement, recommendation, or favoring by the United States Government or any agency thereof. The views and opinions of authors expressed herein do not necessarily state or reflect those of the United States Government or any agency thereof. 


\section{DISCLAMMER}

Portions of this document may be illegible in electronic image products. Images are produced from the best available original document. 
